\title{
Levitation force analysis of medium and low speed maglev vehicles
}

\author{
Guoqing LIU*, Yin CHEN \\ Key Laboratory of Magnetic Levitation Technologies and Maglev Trains (Ministry of Education of China), School of Electrical \\ Engineering, Southwest Jiaotong University, Chengdu 610031, China
}

\begin{abstract}
The rule of levitation force variation with different structure and electromagnetic parameters provides a basis for electromagnet design of electromagnetic suspension (EMS) medium and low speed maglev vehicles. In order to acquire accurate calculation results of levitation force, different calculation methods, including analytical method, 2D FEM (finite element method), and 3D FEM, are applied to investigate the impact of various structural parameters, such as excitation current, air gap, lateral offset, and pole width, on levitation force. The analytical analysis is based on the classic mathematical model of levitation force between electromagnet and rail and performed with MATLAB. In the 2D and 3D FEMs, the numerical calculation of the levitation force is conducted with Ansoft by taking the magnetic saturation into account. In addition, the longitudinal end effect on the levitation force calculation is considered in the 3D FEM. The results show that the 3D FEM is the most accurate among the above three methods for calculating the levitation force, and the analytical method can only work for small current and/or large air gap conditions. A lateraloffset between vehicle and rail will reduce the levitation force; the levitation force descends sharply once the lateral offset exceeds the threshold, i.e., $8 \%$ of the pole width for U-shaped electromagnets. The maximum lift-to-weight ratio emerges when the pole width ratio of $F$ type rail to electromagnet is 6:7. This may offer a reference for EMS maglev vehicle design and application.
\end{abstract}

Key words: EMS; levitation force; analytical solution; finite element method; electromagnet design

(C) 2012 JMT. All rights reserved.

\section{Introduction}

$\mathrm{M}$ edium and low speed maglev vehicles provide an important alternative for future urban traffic because of their outstanding advantages, such as low noise, high speed, and pollution-free nature. At present, Japanese high speed surface transport (HSST) [1-3] is considered to have the most mature medium and low speed maglev plans in the world. The suspension mechanism of HSST is electromagnetic suspension (EMS). The vertical levitation force and the horizontal lateral force are generated by the interaction between electromagnet and rail. As an important part of maglev vehicles, the electromagnet influences overall per-formance of maglev vehicles, such as dead weight, power, stability, and rail cost. Accurately calculating the levitation force of the electromagnet is a basis for magnet design.

To date, most studies of HSST in the world focused on the control system [4-6] and little attention was directed to the characteristics of levitation force. Although

Received May 14, 2012; revision accepted May 29, 2012

*Corresponding author. E-mail: 034115@163.com (G.Q. LIU)

(C) 2012 JMT. All rights reserved

doi: 10.3969/j.issn.2095-087X.2012.02.006
Yin and Chen [7] performed analytical calculation of levitation force, magnetic saturation was not considered in their work, which would reduce the reliability of the calculation result. $\mathrm{Hu}[8]$ calculated the levitation force of the EMS maglev train using the theory of magnetic circuits, but his method was just an approximation. The result was mainly about the dynamic performance of the maglev train and the impact of structural parameters on levitation force was not analyzed in detail. Zhang and Li [9] studied the impact of rail curvature on levitation force, but the electromagnet design parameters were not discussed. Generally, the studies of EMS maglev vehicle levitation force in China are rarely reported, and there is a lack of adequate fundamental theories for electromagnet design.

In this paper, we calculate the levitation force of HSST comprehensively using analytical solution, 2D FEM (finite element method), and 3D FEM, separately. Then, the difference of the calculation results among different methods and the causes leading to the difference are analyzed. Taking magnetic saturation and longitudinal end effect into account, the 3D FEM produces the levitation force and lift-to-weight ratio corresponding to different parameters, such as lateral offset, electro-magnet structural parameters, and excitation source 
parameters. Based on the characteristics of levitation force, an optimal design of electromagnet by 3D FEM is proposed, and the optimal pole width ratio of rail to electromagnet for the maximum lift-to-weight ratio is obtained. This may provide guidance for electromagnet design in engineering applications.

\section{The analytical model}

The HSST vehicle has an independent levitation system composed of a U-shaped electromagnet and an inverted U-shaped steel rail as shown in Fig. 1. An attractive force between the electromagnet and steel rail is generated when current passes through the coil. The electromagnet is then attracted to the fixed rail [10-12]. If the current in the electromagnet increases, the attractive force also increases. Therefore, when enough energy is put into the electromagnets, the vehicle can be kept hovering around the rail.

Table 1 provides the levitation system size parameters of a representative medium and low speed maglev vehicle in China.

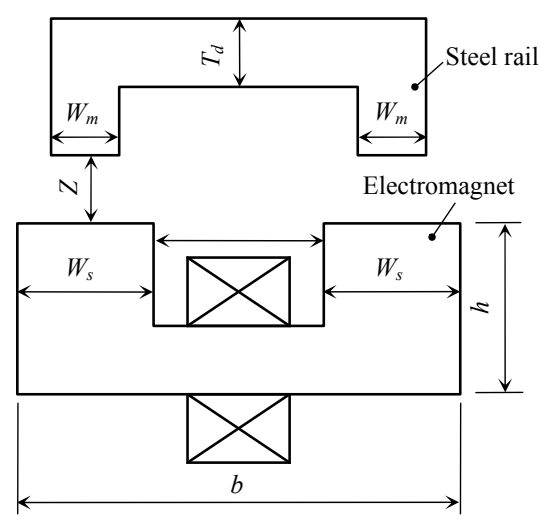

Fig. 1 Steel rail and electromagnet of EMS vehicle

Table 1 Size parameters of the levitation system

\begin{tabular}{lcc}
\hline \multicolumn{1}{c}{ Parameter } & Symbol & Value (mm) \\
\hline Pole width of steel rail & $W_{m}$ & 28 \\
Pole width of electromagnet & $W_{s}$ & 35 \\
Yoke height of steel rail & $T_{d}$ & 28 \\
Yoke height of electromagnet & $h$ & 128 \\
Window width of electromagnet & $W$ & 164 \\
Air gap & $Z$ & 8 \\
Longitudinal length of & $L$ & 1000 \\
$\cdot$ & $b$ & 234 \\
Width of electromagnet & &
\end{tabular}

When the air gap is very small, the air gap magnet field can be treated as a uniform magnetic field and flux is perpendicular to the rail and sub-surface. Magnetic pole width of suspension rail is equal to the electromagnet. In this case, the calculation formula of levitation force can be expressed as follows [7]:

$$
\begin{aligned}
& F_{0}=\frac{B^{2} W_{s}}{\mu_{0}} L, \\
& B=\frac{\mu_{0} \mu N I}{2 Z \mu+\mu_{0}\left[2 h+\left(1+W_{s} / T_{d}\right) b\right]},
\end{aligned}
$$

where $F_{0}$ is the levitation force; $B$ is magnetic flux density in Tesla $(\mathrm{T}) ; \mu_{0}$ is the magnetic permeability of vacuum; $\mu$ is the magnetic permeability of ferromagnetic materials; and other parameters are shown in Table 1.

However, the pole width of the electromagnet is unequal to that of the rail in practical engineering applications, and the lateral offset of the electromagnet with respect to the center of the rail must be considered [4]. An amendment is needed to resolve the formula, which can be expressed as formula (3):

$$
\begin{gathered}
F_{z}=F_{0}\left[1+\frac{2 Z}{\pi W_{m}}+\frac{\beta-d}{\pi W_{m}} \arctan \left(\frac{Z}{\beta-d}\right)+\right. \\
\left.\frac{\beta+d}{\pi W_{m}} \arctan \left(\frac{Z}{\beta+d}\right)\right],
\end{gathered}
$$

where $d$ is the lateral offset of the electromagnet with respect to the rail center, and $\beta$ is given by

$$
\beta=\frac{W_{s}-W_{m}}{2} .
$$

To obtain the levitation force, an M-file is created in MATLAB. The file can be programmed with codes based on formula (3). The analytical calculation is performed with MATLAB. Detailed solution procedure can be found in reference [13].

\section{The finite element models}

Levitation forces are computed by Ansoft Maxwell based on Maxwell's equations in differential form [14].

First, an accurate 2D model of the levitation system is built using AutoCAD, saved as *.dxf file, and then imported into Ansoft (Fig. 2(a)). Considering that it is difficult to build a 3D model in Ansoft directly, the 3D model of the levitation system is built with SolidWorks, and is exported as a *.sat file. By creating a $3 \mathrm{D}$ project and importing the *.sat file, we obtain a useful 3D model in Ansoft as shown in Fig. 2(b). 


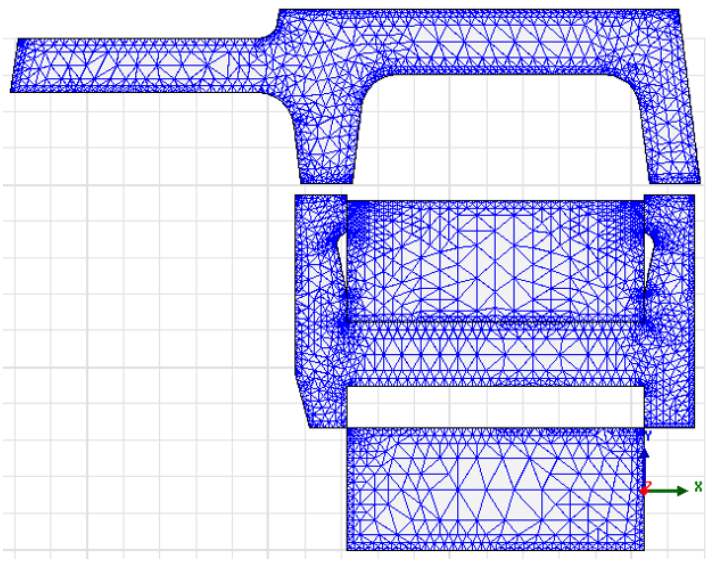

(a) 2D model

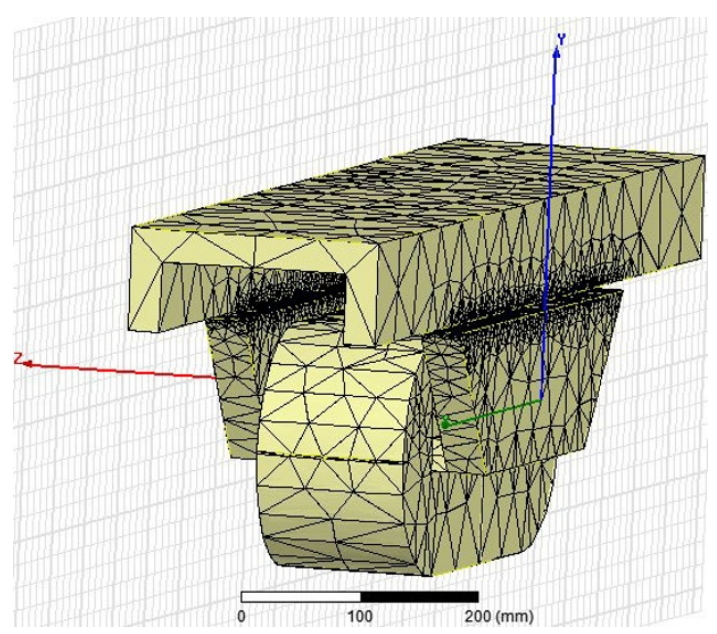

(b) $3 \mathrm{D}$ model

Fig. 2 Finite element models of the levitation system in Ansoft

The material of the electromagnet and the steel rail is steel 10 in accordance with the executive standard GB/T699-1999. The $B-H$ curve of steel 10 is shown in Fig. 3, where $B$ is the magnetic flux density and $H$ is the magnetic field intensity. We define the material by inputting its $B-H$ curve points in Ansoft [15].

The balloon boundary condition is assigned to the models, and current excitation is selected. This boundary is considered to be far away from the other sources in the problem. Then, an adaptive analysis is performed as shown in Fig. 4. Finally, we obtain the calculation results by performing the solution process.

It is easy to modify the excitation source, air gap, lateral-offset, and pole width of the electromagnet in the model in Ansoft. When a model parameter is changed, the solution process needs to run again to generate the new result. By changing the value of a particular structural parameter and keeping the other parameters unchanged, we can obtain the relationship curves between levitation force and the parameters of the levitation system.

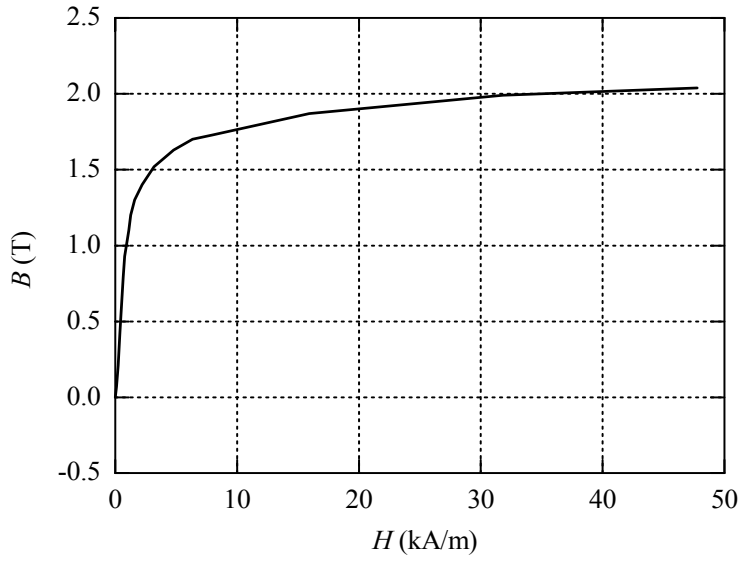

Fig. $3 B$ - $H$ curve of steel 10

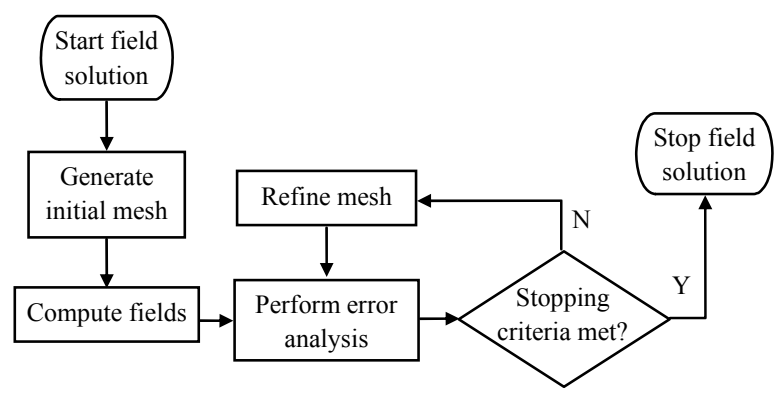

Fig. 4 Flow chart of adaptive analysis

\section{The impacts of structural parameters on levitation force}

\subsection{Effect of excitation current on the levitation force}

The relationship curves between levitation force and ampere turns for the three methods are shown in Fig. 5. It can be seen that when the excitation current source takes a small value (the ampere turns is less than $7 \mathrm{kA}$ ), the analytical results are consistent with those of $2 \mathrm{D} / 3 \mathrm{D}$ FEMs. With increasing ampere turns greater than $7 \mathrm{kA}$,

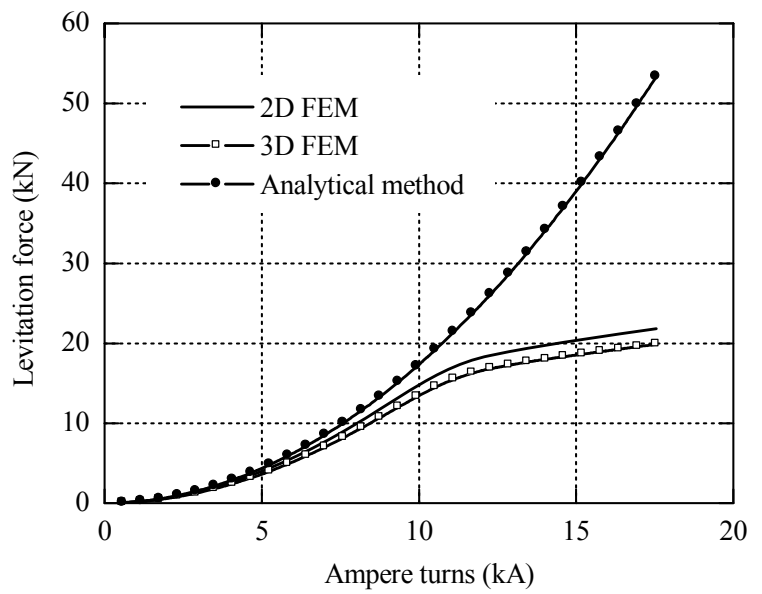

Fig. 5 Levitation force vs ampere turns for analytical and FEA solutions 
the analytical results gradually become larger than those obtained from finite element methods. The main reason is that the nonlinearity of the magnetic material permeability cannot be covered by formula (3).

The 2D FEM can not analyze the longitudinal end effect as the 3D FEM does; therefore, there is a difference of about $8 \%$ between the $2 \mathrm{D}$ and $3 \mathrm{D}$ FEA results.

\subsection{Effect of air gap on the levitation force}

The relationship curves between levitation force and air gap are shown in Fig. 6. We can see from Fig. 6 that the three lines tend to agree with each other when the air gap is greater than $8 \mathrm{~mm}$. However, with decreasing air gap, the analytical results become increasingly larger than those obtained from the FEMs. This is because the analytical method can not take the magnetic saturation into consideration. As the air gap becomes smaller, the core of the electromagnet is easier to be saturated.

As a key factor, even a slight change of air gap can also cause a significant change of the levitation force. If the other structural parameters are kept unchanged, the levitation force will increase in inverse proportion to the air gap value, but still with a large change rate, indicating that the levitation force is difficult to control. On the other hand, the change rate will reduce as air gap value increases. In this case, however, the levitation force is small. Therefore, an appropriate value of air gap, i.e. $8 \mathrm{~mm}$, is selected as the rated value.

\subsection{Effect of lateral offset between vehicle and rail on the levitation force}

When the maglev vehicle runs through a curve, the levitation force will be reduced because of a lateral offset between vehicle and rail. Fig. 7 shows the variation rule of levitation force with lateral offsets from 0 to $14 \mathrm{~mm}$.

It can be seen from Fig. 7 that when the whole magnetic pole of the electromagnet is located just beneath the magnetic pole of the rail, i.e., the lateral offset is zero, the levitation force remains unchanged. With an increase of the lateral offset, the magnetic pole of the electromagnet is partially located beneath the magnetic pole of the rail, and the levitation force declines conspicuously. Compared with the result of 3D FEM, the analytical formula has a maximum relative error of about $5.5 \%$.

When the value of the lateral offset increases to about $2.2 \mathrm{~mm}$ (i.e., $8 \%$ of the electromagnet pole width), the levitation force reduces only $1 \%$. However, with further increasing values of lateral offset, the levitation force begins to descend more rapidly; e.g., a lateral-offset of $50 \%$ pole width causes a $16 \%$ descent in levitation force. Therefore, $8 \%$ of the electromagnet pole width is a turning point, and the lateral offset should be controlled

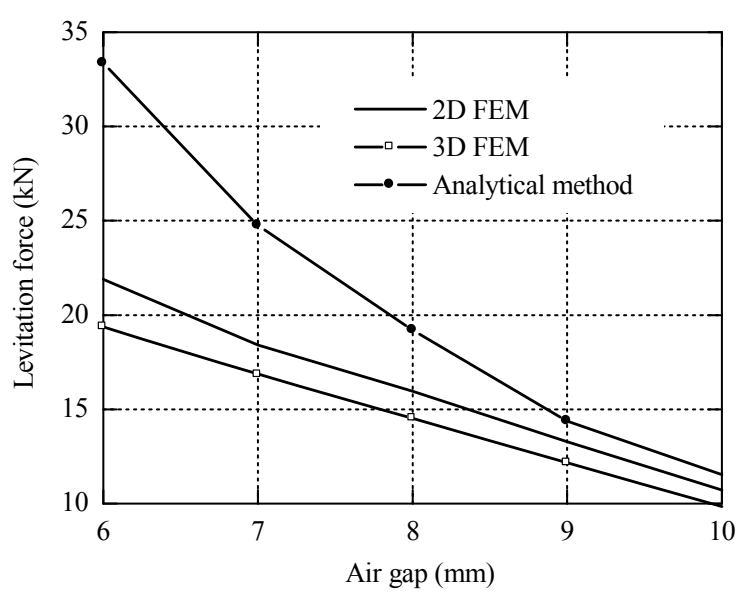

Fig. 6 Variation rule of levitation force with air gap

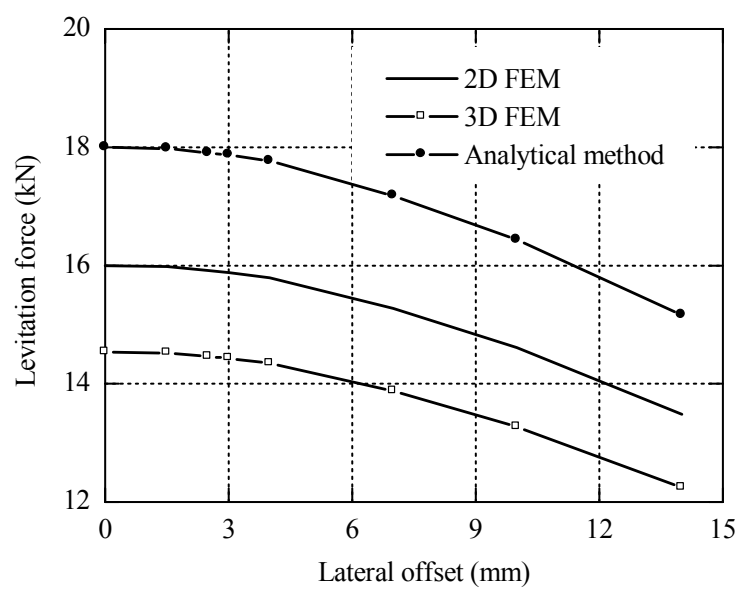

Fig. 7 Variation rule of levitation force with lateral offset

within this limit. From Fig. 7, the levitation force calculated by 3D FEM are the smallest in any case because of the longitudinal end effect of the electromagnet. Compared with the other two methods, the 3D FEM has covered not only the magnetic saturation but also the longitudinal end effect of the electromagnet, and hence produces more reasonable results.

\subsection{Effect of electromagnet magnetic pole width on lift-to-weight ratio}

Lift-to-weight ratio $k$ is an important indicator for the evaluation of the levitation force, and is given by

$$
k=F / G,
$$

where $F$ is levitation force, and $G$ is weight of electromagnet. Lift-to-weight ratio reflects the vehicle weight suspended by per unit weight of the electromagnet. Generally, a larger lift-to-weight ratio indicates better levitation performance. Gradually increasing the pole width of the electromagnet from 14 to $33 \mathrm{~mm}$ and keeping the other structural parameters unchanged in the above three models, we can first obtain the levitation force results, and then generate the lift-to-weight ratio 


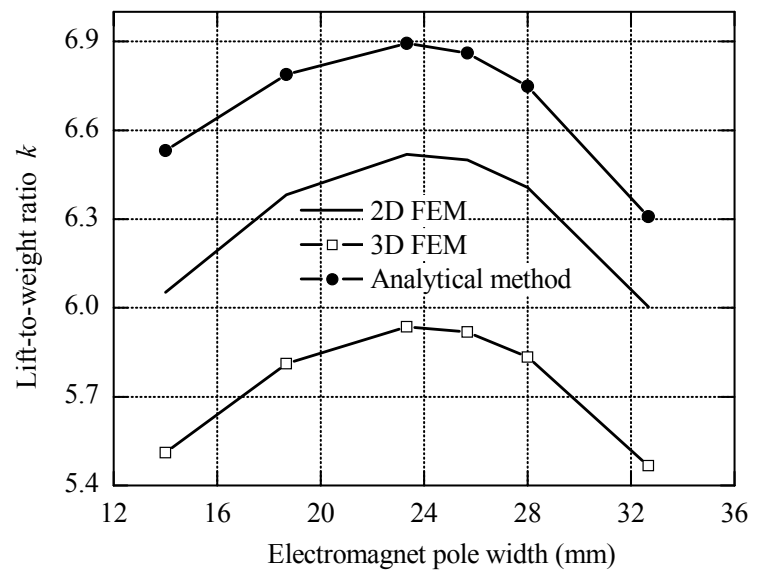

Fig. 8 Variation rule of Lift-to-weight ratio with electromagnet pole width

by dividing the results by the weight of the electromagnet (Fig. 8).

As Fig. 8 shows, the lift-to-weight ratio first increases and then decreases with increasing electromagnet magnetic pole width. The highest buoyant weight ratio occurs when the electromagnet magnetic pole width is $24 \mathrm{~mm}$, i.e., the pole width ratio of electromagnet to steel rail is 6:7. Therefore, wider electromagnet magnetic pole does not mean better performance, and a proper electromagnet pole width should be determined in designing the electromagnet.

\section{Conclusions}

In this paper, three methods, including the analytical method, 2D FEM, and 3D FEM, are applied to analyze the levitation force characteristics of EMS medium and low speed maglev vehicles under different electromagnetic parameters and structure parameters. Conclusions can be drawn as follows:

(1) In the condition of low current or large air gap, the analytical formula has been proven to have a high accuracy, and the difference of calculation results between analytical solution and FEM is not more than $7 \%$. With increasing current or decreasing air gap, however, the analytical method becomes inaccurate. The reason is that the magnetic saturation of the core cannot be considered in the analytical method.

(2) A lateral-offset between vehicle and rail will reduce the levitation force. A small lateral-offset can hardly change the levitation force. However, levitation force degrades rapidly when lateral-offset exceeds a certain value, i.e., $8 \%$ of pole width for U-shaped electromagnets.

(3) Increasing the pole width of the electromagnet leads to not only the increase of the levitation force but also the weight. Therefore, what should be considered in electromagnet design is both levitation and gravity. The maxi- mum lift-to-weight ratio emerges when the pole width ratio of $\mathrm{F}$ type rail to electromagnet is 6:7.

(4) Taking magnetic saturation, transverse and longitudinal end effects into account, the 3D FEM can obtain more accurate calculation results of levitation force.

\section{References}

[1] J.Q. Wang, O. Fujiwara, S. Ishimoto, et al., Electromagnetic environment assessment for the Tobu-Kyuryo-Line (HSST System) in Japan, In: Maglev 2004 Proceedings, Shanghai, China, 2004: 636-639.

[2] Y. Yasuda, M. Fujino, M. Tanaka, et al., The first HSST maglev commercial train in Japan, In: Maglev 2004 Proceedings, Shanghai, China, 2004:76-85.

[3] T. Michio, G. Kwok, K. Kubota, Marketing strategy of the HSST system, In: The 19th International Conference on Magnetically Levitated Systems and Linear Drives, Dresden, Germany, 2006: 1-5.

[4] K.F. Zhu, J.W. Dong, X.G. Lei, et al., The current minimum variance control in the electromagnetic suction suspension systems, Electric Switchgear, 2010, 48(1): 58-60 (in Chinese).

[5] Y.K. Li, J. Li, Suspension control of low-speed maglev train based on kalman filter, Computer Simulation, 2011, 28(5): 346-375 (in Chinese).

[6] F. Cao, Comparison and selection of control method for mid-low speed maglev train, Science \& Technology Information, 2010(3): $432-417$ (in Chinese).

[7] L.M. Yin, G.R. Chen, Levitation electromagnet design principles and calculation methods for Suction-type levitation train, Electric Drive for Locomotives, 1992(5): 1130 (in Chinese).

[8] J.S. Hu, Analysis of suspension force of the EMS maglev train, Journal of Southwest Jiaotong University, 2001, 36(1): 44-47 (in Chinese).

[9] G. Zhang, J. Li. Calculation of electromagnetic force of low-speed maglev on a vertical curve, Proceedings of the CSEE, 2012, 32(3): 150-156 (in Chinese).

[10] M. Murai, M. Tanaka, Magnetic levitation (maglev) technologies, Japan Railway \& Transport Review, 2000(25): 61-67.

[11] P.K. Biswas, S. Bannerjee, Analysis of U-I and U-U type rail and actuator used in electromagnetic levitation system using FEM software, International Journal of Emerging Technology and Advanced Engineering, 2012, 2(5): 32-39.

[12] Y.H. Tzeng, T.C. Wang, Optimal design of the electromagnetic levitation with permanent and electro magnets. IEEE Trans. Magn, 1994, 30(6): 4731-4733.

[13] S.J. Chapman, MATLAB programming for Engineers, New York: Wadsworth Group Press, 2002.

[14] J. Hoffmann, C. Hafner, P. Leidenberger, et al., Comparison of electromagnetic field solvers for the $3 \mathrm{D}$ analysis of plasmonic nano antennas, In: Proc. SPIE, Munich, Germany, 2009: 0-11.

[15] A. Kakilli, Magnetic analysis of modified-rotor switched reluctance motor, Electronics and Electrical Engineering, 2011, 115(9): 21-26. 